\title{
Photometric follow-up observation of some SuperWASP transiting planet candidates
}

\author{
Sheng-hong $\mathrm{Gu}^{1}$, Andrew Collier Cameron ${ }^{2}$, Xiao-bin Wang ${ }^{1}$, Li-yun \\ Zhang $^{1}$, Xiang-song Fang ${ }^{1}$ and Xue-jing $\mathbf{L i}^{1}$ \\ ${ }^{1}$ National Astronomical Observatories/Yunnan Observatory, CAS, \\ Kunming 650011, China \\ email: shenghonggu@ynao.ac.cn \\ ${ }^{2}$ School of Physics and Astronomy, University of St Andrews, \\ Fife KY16 9SS, UK \\ email: acc4@st-and.ac.uk
}

\begin{abstract}
Three SuperWASP transiting planet candidates were observed through R or I filters using the 1-meter telescope and CCD camera of Yunnan Observatory from 2006 to 2007. The relative photometric data were corrected for the systematic errors by means of Tamuz et al. (2005) and Collier Cameron et al. (2006)'s algorithms. The resulting light curves demonstrate that one of three targets is a potential exoplanet candidate, which is worthy to perform further follow-up observation to clarify.
\end{abstract}

Keywords. exoplanet, transit, photometry

\section{Introduction}

The SuperWASP project (Pollacco et al. 2006) is an UK wide-field survey for exoplanet transits. Since this project was started in 2004, many candidates have been discovered by the WASP consortium (Christian et al. 2006, Clarkson et al. 2007, Collier Cameron et al. 2007, Lister et al. 2007, Street et al. 2007). Due to the small apertures of the SuperWASP telescopes, it is very important to make follow-up photometry for these candidates by using telescopes with larger apertures. Thus, we began a follow-up observation project at the $1.0 \mathrm{~m}$ telescope of Yunnan Observatory in 2006. Up to now, we have finished photometric observations for 3 SuperWASP targets: One of them was already classified to be a SB1 binary (Collier Cameron et al. 2007); for the second one, we could not find the light variation; we found a transit-like light curve for the third candidate with a magnitude of 9.37 in $\mathrm{V}$ band. Here, we present the results of candidate 3.

\section{Observation}

We performed the photometric observation for candidate 3 of our SuperWASP targets using the 1.0m telescope at Yunnan Observatory on September 18, 2007. The equipment used in the observation is a $1 \mathrm{k} \times 1 \mathrm{k}$ CCD camera with the I filter. The field of view is about $6^{\prime} .5 \times 6^{\prime} .5$. The exposure time is between 20 and 40 seconds depending on the observing condition.

\section{Data reduction}

The observed images are reduced with the IRAF package, including the bias subtraction, flat-fielding and cosmic ray removal. The target star and 5 comparisons in the 


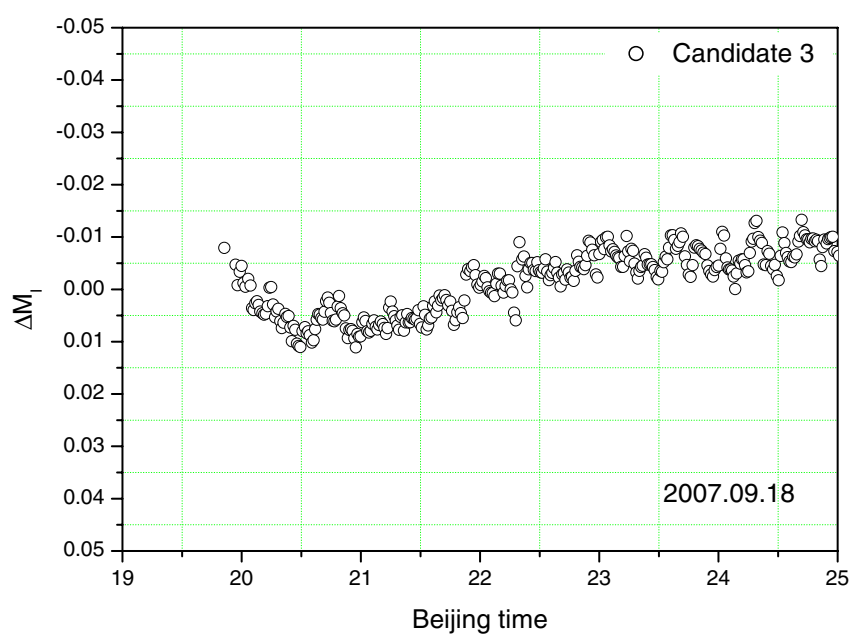

Figure 1. The reduced light curve of candidate 3.

same field of view are measured by using the APPHOT sub-package of IRAF through an aperture with radius of 12.5 ". The result light curve consists of 500 data points.

It is well-known that the transit depth of exoplanets is very shallow. In order to hunt the transit signal, we need to use some new methods to improve the quality of the observed light curve. Here, we have utilized the algorithms of Tamuz et al. (2005) and Collier Cameron et al. (2006) to remove the systematic effects in photometric data so as to obtain the final light curve with high quality.

\section{Preliminary result}

Fig. 1 shows the final light curve for candidate 3 on Sept. 18, 2007 after removing the systematic effects. This is potentially a transit-like light curve, it is worthy to do further follow-up observations to confirm. From this light curve, we can find that the transit center time deviates from the ephemeris formulae provided by the SuperWASP consortium. In our observation, the transit center time is coming earlier by several ten minutes than the prediction, which suggests that the orbital period derived before is a little longer.

We shall carry out further photometric and spectroscopic observations in this winter to clarify whether the candidate 3 of our samples is an exoplanet.

\section{Acknowledgements}

This work is partly supported by NSFC under grant No. 10673027.

\section{References}

Christian, D. J. et al. 2006, MNRAS, 372, 1117

Clarkson, W. I. et al. 2007, MNRAS, 381, 851

Collier Cameron, A. et al. 2006, MNRAS, 373, 799

Collier Cameron, A. et al. 2007, MNRAS, 380, 1230

Lister, T. A. et al. 2007, MNRAS, 379, 647

Pollacco, D. L. et al. 2006, PASP, 118, 1407

Street, R. A. et al. 2007, MNRAS, 379, 816

Tamuz, O., Mazeh, T. \& Zucker, S. 2005, MNRAS, 356, 1466 